\title{
Introduction by the Editors
}

Sbek thom (Large [Leather] Hide) is the name of a genre of Khmer oral musical theater, a complex of shadow plays that draw their characters and stories from the Khmer version of the Indian Ramayana. Prior to the recent war, sbek thom performances were common in some areas of Cambodia. Pech Tum Kravel singlehandedly spent much time and energy researching thoroughly every available document on Khmer shadow theater, assembled photographs of one extant collection of shadow figures, and produced an unprecedented manuscript on the genre. It is quite an extensive work, though it is only preliminary, in the sense that the organization of the text is not sequential and sometimes rather desultory. Moreover, some of the observations about the history of sbek thom need to be confirmed, and there is much more that can be said about the place of the genre in Khmer history and culture, including a reasoned description and analysis of the historical relationships between this art form and various genres of Thai shadow theater. Still, the work is impressive and essential, especially now, as a tool for teaching about Khmer life and as a means to preserve and document what is known now about the Khmer performing arts. We offer it as a stimulus to work on the Khmer arts in Khmer and other languages, especially by young Khmer scholars.

The English version of this work is a free translation. The editor has deleted words, phrases, and sometimes whole sentences of the Khmer, and altered many others, to make the work more readily accessible to a general audience.

In transliterating the Khmer script, we have followed the past practices of French and English language writers on Cambodian performing arts, including those who produced the volume entitled Traditional Music Instruments of Cambodia, published by UNESCO in 1994. Though more consistent (and scientific) methods of representing Khmer now exist, we have chosen to place this work in the mainstream of past scholarship, and thereby permit more ready associations between the Khmer place names, objects, and dramatic characters discussed in this work and their counterparts in previous publications. 
The preparation of this book from the manuscript was accomplished in several stages. From June to September 1994, the Khmer manuscript was typed and preliminary editing done by Thavro Phim. His work was funded in part by a grant to Cornell University's Southeast Asia Program from the Asian Cultural Council (New York, New York). From September 1994 to April 1995, Sos Kem edited the Khmer manuscript and translated the text and captions into English. His work was partly funded by a grant to the Southeast Asia Program from the MFH Fund for Religious, Charitable, and Educational Purposes. From June 1994 until June 1995, Martin Hatch abridged, adapted, and edited the English version and prepared the entire manuscript for its printing in Phnom Penh. The United Nations Educational, Scientific and Cultural Organization (UNESCO), through its offices in Phnom Penh, arranged and provided some of the funding for the printing of the entire work. The Japan Sotoshu Relief Committee (Phnom Penh office) underwrote a significant portion of the costs of printing. The entire Phnom Penh aspect of the project was coordinated and executed by Keiko Miura (Assistant Culture Programme Officer in Phnom Penh) and Khun Khuon (UNESCO, Phnom Penh).

Ithaca, New York: June 1995

Sos Kem and Martin Hatch 